\title{
Jacaric Acid is Rapidly Metabolized to Conjugated Linoleic Acid in Rats
}

\author{
Ryo Kijima', Taro Honma', Junya Ito', Masao Yamasaki², Aya lkezaki², \\ Chihiro Motonaga ${ }^{2}$, Kazuo Nishiyama ${ }^{2}$ and Tsuyoshi Tsuduki ${ }^{1 *}$ \\ ${ }^{1}$ Laboratory of Food and Biomolecular Science, Graduate School of Agriculture, Tohoku University (1-1 Tsutsumidori amamiyamachi, Aobaku, \\ Sendai 981-8555, JAPAN) \\ ${ }^{2}$ Department of Biochemistry and Applied Biosciences, University of Miyazaki (1-1 Gakuen-kibanadai-nishi, Miyazaki 889-2192, JAPAN)
}

\begin{abstract}
We have shown previously that jacaric acid (JA; 8c,10t,12c-18:3), which has a conjugated triene system, has a strong anti-tumor effect. However, the characteristics of absorption and metabolism of JA have yet to be determined in vivo, and the details of absorption and metabolism of JA in the small intestine are particularly unclear. This information is required for effective use of $\mathrm{JA}$ in humans. Therefore, in this study we examined absorption and metabolism of JA using cannulation of the thoracic duct in rats. Emulsions of two test oils, jacaranda seed oil and tung oil, which contain JA and $\alpha$-eleostearic acid ( $\alpha$-ESA; $9 c, 11 t, 13 t-18: 3)$, respectively, were administered to rats and lymph from the thoracic duct was collected over $24 \mathrm{~h}$. We examined the rate of absorption of JA and possible conversion to a conjugated linoleic acid (CLA) containing a conjugated diene system. The positional isomerism of the CLA produced by JA metabolism was determined using gas chromatography-electron impact/mass spectrometry. The rate of absorption and percentage conversion of JA were compared with those of $\alpha$-ESA. We found that JA is rapidly absorbed and converted to a CLA in rats and that the percentage conversion of JA was lower than that of $\alpha$-ESA. This is the first report on the absorption and metabolism of $\mathrm{JA}$ and this information may be important for application of $\mathrm{JA}$ as a functional food.
\end{abstract}

Key words: conjugated fatty acid, conjugated linolenic acid, conjugated linoleic acid, jacaric acid, lymph

\section{INTRODUCTION}

Conjugated fatty acid is a generic term used for fatty acids with conjugated double bonds, as exemplified by conjugated linoleic acid $(\mathrm{CLA})^{1}$. There are several CLA isomers due to positional and geometrical isomerism of the conjugated double bonds, and the major naturally-occurring CLA isomer is referred to as 9c,11t-18:2 (Fig. 1) ${ }^{1)}$. CLAs were first reported to have an anti-tumor effect, and subsequently various physiological effects have been shown, including a role in regulation of lipid metabolism ${ }^{1-5)}$. These CLA activities are associated with the conjugated double bond system. CLAs are found naturally and are especially present in ruminant fats such as beef tallow and milk fat ${ }^{1}$. However, the CLA level in these foodstuffs is around $1 \%(\mathrm{w} / \mathrm{w})$, which prevents use of the natural fats as health-promoting foods containing CLAs. Therefore, oils that include CLAs are currently prepared from alkali-isomerization of vegetable oils such as safflower oil and these products are marketed as health supplements ${ }^{6,7)}$.
Conjugated fatty acids other than CLAs also exist in nature, including seed oils of plants that include conjugated triene fatty acids such as $\alpha$-eleostearic acid ( $\alpha$-ESA; 9c,11t,13t-18:3, Fig. 1) and jacaric acid (JA; 8c,10t,12c18:3, Fig. 1) at levels of $30 \%$ to $80 \%(\mathrm{w} / \mathrm{w})^{8-10)}$. We are particularly interested in seed oils including conjugated linolenic acids (CLnAs), which are the only conjugated fatty acids that can be prepared from natural sources in bulk, and we have previously shown that $\alpha$-ESA (which is a CLnA) has a stronger anti-tumor effect than CLA in vitro and in vivo ${ }^{11)}$. $\alpha$-ESA also has useful physiological effects and we have shown that the conjugated triene in $\alpha$-ESA is partially saturated at the $\Delta 13$ position and converted to 9c,11t-CLA, which has a conjugated diene, in rats. The $\Delta 13$ saturation is an NADPH-dependent enzymatic reaction that occurs most rapidly in rat liver and small intestine $^{12-14)}$.

In our recent studies, we showed that the anti-tumor activity of CLnAs is stronger than that of CLAs, and that JA

\footnotetext{
*Correspondence to: Tsuyoshi Tsuduki, Laboratory of Food and Biomolecular Science, Graduate School of Agricultural Science, Tohoku University, 1-1 Tsutsumidori-Amamiyamachi, Aoba-ku, Sendai 981-8555, JAPAN

E-mail: tsudukit@m.tohoku.ac.jp

Accepted December 18, 2012 (received for review November 20, 2012)

Journal of Oleo Science ISSN 1345-8957 print / ISSN 1347-3352 online

http://www.jstage.jst.go.jp/browse/jos/ http://mc.manusriptcentral.com/jjocs
} 


\section{R. Kijima and T. Tsuduki et al.}

had the strongest effect among CLnAs ${ }^{15)}$. JA is a natural product that is present in jacaranda seed ${ }^{10)}$ and may have applications in humans as a useful physiologically active fatty acid. However, the characteristics of absorption and metabolism of JA have yet to be determined in vivo, particularly in the small intestine. This information is very important for effective use of JA in humans. Additionally, it is of interest to determine whether the conjugated triene system of JA undergoes a saturation reaction in vivo. We are interested in this reaction because it is a rare example of fatty acid metabolism involving saturation of a double bond. For these reasons, we investigated the absorption and metabolism of JA in rat intestine using a previously described lipid absorption assay based on collection of lymph from the thoracic duct ${ }^{13,16}$.

The absorption and metabolism of JA in rat intestine were examined using two test oils: jacaranda seed oil (JSO), which contains a triacylglycerol (TG) form of JA; and tung oil (TO), which contains a TG form of $\alpha$-ESA, an isomer of JA. First, we examined whether JA was absorbed in rats. To determine if JA is converted to a CLA, the structure of the metabolite was determined using gas chromatography-electron impact/mass spectrometry (GC-EI/MS) ${ }^{12,13)}$. The rate of absorption and conversion rate of JA were then compared with those of $\alpha$-ESA. The results showed that JA is rapidly absorbed and converted to a CLA in rats and that the percentage conversion of JA is lower than that of $\alpha$-ESA. This is the first report on the absorption and metabolism of JA and the findings may be useful for application of JA as a functional food.

\section{EXPERIMENTAL PROCEDURES}

\subsection{Materials}

Trimethylsilyldiazomethane (10\% in hexane, v/v) was purchased from GL Sciences, Inc. (Tokyo, Japan). Sodium methoxide/methanol ( $1 \mathrm{mmol} / \mathrm{L}$ solutions) was purchased from Wako Pure Chemicals Industries (Osaka, Japan). JSO was a kind gift from the Miyazaki Prefectural Government (Miyazaki, Japan). TO was kindly provided by The Nisshin OilliO Group (Tokyo, Japan). JA (99\% purity) and $\alpha$-ESA (99\% purity) were purchased from Larodan Fine Chemicals AB (Malmö, Sweden). 9c,11t-CLA (98\% purity) was obtained from Cayman Chemical Co. (Ann Arbor, MI).

\subsection{Methods}

2.2.1 Animals and treatments

All procedures were performed in accordance with the Animal Experiment Guidelines of Tohoku University. The animal protocol was approved by the Animal Use Committee at Tohoku University.

2.2.2 Cannulation of the thoracic duct of rats

Operations and maintenance of rats and all other proce- dures were performed as described previously ${ }^{13,16)}$. Nineweek-old male Sprague-Dawley rats were obtained from CLEA Japan (Tokyo, Japan). After acclimatization with MF Standard Rodent Chow (Oriental Yeast) and distilled water (free access) for 1 week, a cannula (SV35, Dural Plastics, Tokyo, Japan) was inserted into the left thoracic channel to collect lymphatic fluid and a catheter (SP-55, Dural Plastics, Tokyo, Japan) was also inserted into the stomach. After surgery, each rat was placed in a restraining cage in a warm recovery room. A physiological solution containing $139 \mathrm{mmol} / \mathrm{L}$ glucose and $85 \mathrm{mmol} / \mathrm{L} \mathrm{NaCl}$ was continuously infused overnight at a rate of $3 \mathrm{~mL} / \mathrm{h}$ through the stomach cannula. The same solution was also provided as drinking water. The next morning, after collection of lymph for $2 \mathrm{~h}$ as a blank control, the rats were infused with $3 \mathrm{~mL}$ of an emulsion as a single bolus through the stomach catheter. Test emulsions containing $200 \mathrm{mg}$ of the test oils (JSO or TO), $50 \mathrm{mg}$ of fatty acid-free albumin, and $200 \mathrm{mg}$ of sodium taurocholate were prepared by ultrasonication. After infusing the emulsion into the rats, infusion of the glucose $/ \mathrm{NaCl}$ solution was continued. Lymph was collected in an EDTA-containing tube for analysis at the following intervals after test-oil infusion: 0-2, 2-4, 4-6, 6-8 and 8-24 h. After lymph flow was measured, lymph was stored at $30^{\circ} \mathrm{C}$ until analysis.

2.2.3 Gas chromatography (GC) analysis

Total lipids from each lymph sample were extracted using the Bligh \& Dyer procedure ${ }^{17)}$. A volume of $2 \mathrm{~mL}$ of $\mathrm{MeOH}: \mathrm{CHCl}_{3}(2: 1 \mathrm{v} / \mathrm{v})$ containing butylated hydroxytoluene (BHT) was added to $400 \mu \mathrm{L}$ of each lymph sample and mixed by vortex for $2 \mathrm{~min}$. Then $1 \mathrm{~mL}$ of $\mathrm{MeOH}: \mathrm{CHCl}_{3}(1: 1$ $\mathrm{v} / \mathrm{v})$ containing BHT was added, mixed by vortex for $2 \mathrm{~min}$, and centrifuged for $5 \mathrm{~min}$ at $4^{\circ} \mathrm{C}$ and $907 \mathrm{~g}$ (Takara Tomy, Japan). The lower layer was transferred to another tube and $1 \mathrm{~mL}$ of $\mathrm{CHCl}_{3}$ containing BHT was added to the upper layer, mixed by vortex for $2 \mathrm{~min}$, and centrifuged. This step was repeated three times. Total lipids were dried under a stream of nitrogen gas. Total lipids or TO or JSO test oils and a known amount of heptadecanoic acid (17:0) (Sigma, St. Louis, MO) as an internal standard were methylated by addition of trimethylsilyldiazomethane for $30 \mathrm{~min}$ and sodium methoxide/methanol for $10 \mathrm{~min}$ at room temperature to prepare fatty acid methyl esters (FAMEs) ${ }^{13,18)}$. The product was subjected to GC (GC-2014, Shimadzu, Kyoto, Japan) using a flame ionization detector and a Supelco wax-10 fused silica capillary column $(60 \mathrm{~m} \times 0.32 \mathrm{~mm}, 0.25$ $\mu \mathrm{m}$ film thickness, Supelco, Bellefonte, PA) using previously described GC conditions ${ }^{13,14)}$. Peak components were identified by comparing retention times with those of commercial FAMEs (Funakoshi, Tokyo). Reference standards were used to identify JA, $\alpha$-ESA and 9c,11t-CLA ${ }^{14,19)}$. The peak for heptadecanoic acid (17:0) was used as a basis for measuring the levels of fatty acids ${ }^{14,19)}$. The fatty acid compositions of the test oils are shown in Table 1. 
The absorption and metabolism of Jacaric acid

Table 1 Fatty acid composition in test oils.

\begin{tabular}{lcc}
\hline & TO (tung oil) & JSO (jacaranda seed oil) \\
\hline \multicolumn{2}{c}{$(\%)$} \\
$16: 0$ & 8.7 & 7.0 \\
$18: 0$ & 3.5 & 6.1 \\
$18: 1(\mathrm{n}-9)$ & 17.7 & 16.1 \\
18:2 (n-6) & 34.8 & 52.6 \\
CLA (18:2) & - & - \\
18:3 (n-3) & 2.2 & 2.2 \\
JA (8c,10t,12c-18:3) & - & 15.9 \\
$\alpha$-ESA (9c,11t,13t-18:3) & 32.8 & - \\
others & 0.3 & 0.1 \\
\hline
\end{tabular}

FAMEs prepared from test emulsion were analyzed by GC.

- , not detected $(<0.1 \%)$.

\subsubsection{Structure determination of CLA}

To determine the positional isomerism of the CLA produced by JA metabolism, total lipids in the lymph of JSOor TO-treated rats $0-2 \mathrm{~h}$ after administration were identified by analyzing the corresponding fatty acid 4,4-dimethyloxazoline (DMOX) derivatives by GC-EI/MS (GCMSQP5050A, Shimadzu Corporation, Kyoto $)^{13,14)}$. DMOX derivatives were prepared after freeing the fatty acids from total lipids in the lymph. The free fatty acids were mixed with 2-amino-2-methyl-1-propanol in a test tube, which was then purged with nitrogen gas, screw-capped and heated at $170^{\circ} \mathrm{C}$. After $30 \mathrm{~min}$, the tube was cooled and a saturated $\mathrm{NaCl}$ solution and n-hexane were added, followed by vigorous shaking. After centrifugation, the hexane phase was dried under a stream of nitrogen gas and the dried residue was then redissolved in an adequate amount of n-hexane. GC-EI/MS was performed using a GC system (GC-17A, Shimadzu) equipped with a Supelcowax-10 fused silica capillary column coupled to an electron impact mass spectrometer (GCMS-QP5050A, Shimadzu). Helium was used as the carrier gas. The injector temperature was $170^{\circ} \mathrm{C}$. The oven temperature was programmed to match the temperature used in the corresponding GC anal$\mathrm{ysis}^{13,14)}$. The temperature of the ion source was $250^{\circ} \mathrm{C}$. Electron impact mass spectra were recorded at $70 \mathrm{eV}$ and analyzed using the CLASS 5000 data system (Shimadzu). 2.2.5 Statistical analysis

Each result is shown as the mean $\pm \mathrm{SE}$. A Dunnett multiple-comparison test or two-tailed unpaired Student t-test was performed to compare findings between groups. A difference was considered to have significance at $p<0.05$.

\section{RESULTS}

The aim of the study was to determine the absorption

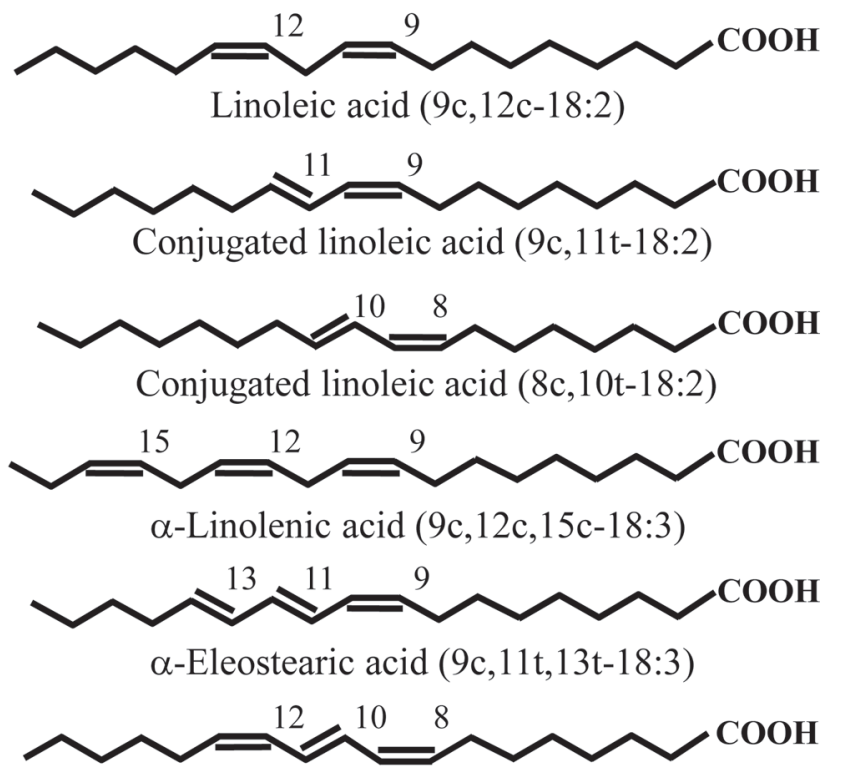

Jacaric acid (8c,10t,12c-18:3)

Fig. 1 Chemical structures of conjugated fatty acids.

and metabolism properties of JA in the rat small intestine. JSO containing the triacylglycerol form of JA was used as the test oil (Table 1) and TO containing the triacylglycerol form of $\alpha$-ESA was used as a comparison oil (Table 1 ). CLA was not detected in JSO or TO (Table 1).

There were no conjugated fatty acids in rat lymph immediately before administration of the oils, based on GC analysis (Fig. 2A). Peaks due to the administered oils appeared in the chromatogram of the lymph $2 \mathrm{~h}$ after administration (Fig. 2B, C). Peaks for $\alpha$-ESA and 9c,11t-CLA appeared in the chromatogram of lymph from rats administered TO (Fig. 2B), showing that $\alpha$-ESA was converted to $9 \mathrm{c}, 11 \mathrm{t}-$ CLA in the rat intestine. Peaks for JA and compound X, which had the same retention time as 9c,11t-CLA, ap- 

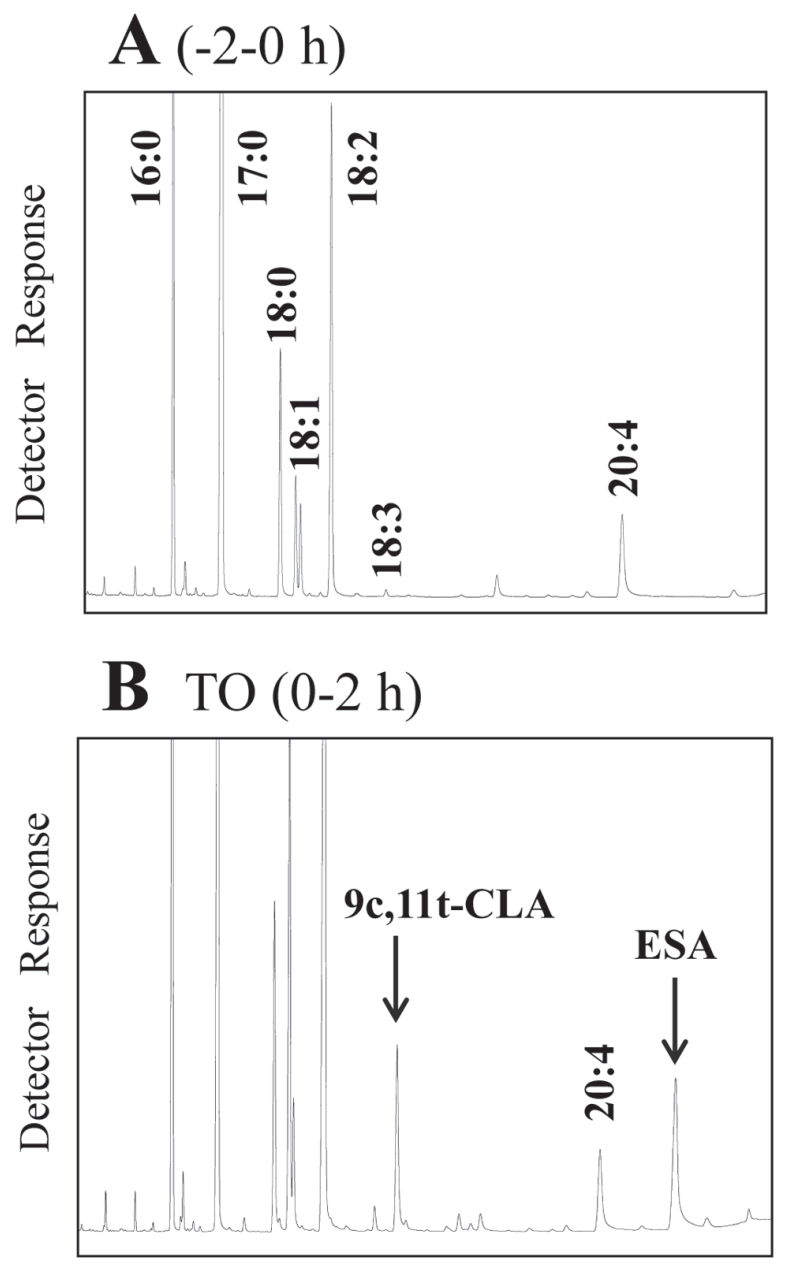

\section{JSO $(0-2 \mathrm{~h})$}

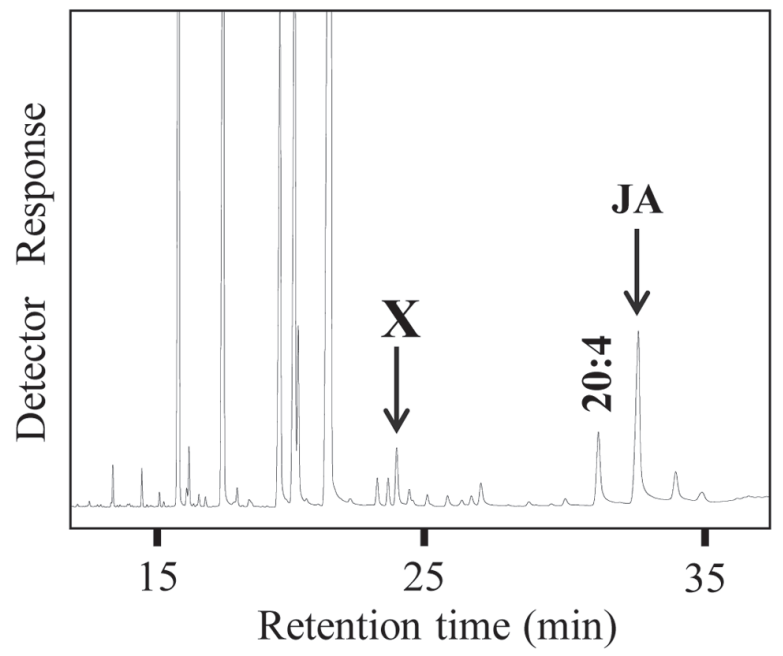

Fig. 2 GC chromatograms of FAMEs synthesized from total lipids in the thoracic duct lymph of rats. Lymph was collected from $-2 \mathrm{~h}$ to $0 \mathrm{~h}$ (A) or from $0 \mathrm{~h}$ to $2 \mathrm{~h}(\mathrm{~B}, \mathrm{C})$ after administration of an emulsion containing TO (B) or JSO (C). peared in the chromatogram of lymph from rats administered JSO (Fig. 2C), suggesting that JA may have been converted to a CLA.

The structure of compound $\mathrm{X}$ formed by JA metabolism was determined using GC-EI/MS. FAMEs of total lipids in lymph from the JSO group were converted to DMOX derivatives and analyzed using GC-EI/MS, in which a fragment due to the presence of conjugated double bond was apparent. The GC-EI/MS spectrum of the DMOX derivative of JA and compound $\mathrm{X}$ in lymph of the JSO group and 9c,11tCLA (standard) had fragments of $\mathrm{m} / \mathrm{z} 113$ and 126, due to fatty acid DMOX derivatives (Fig. 3). The presence of JA in lymph of the JSO group was confirmed by the position of the double bonds (Fig. 3A). The GC-EI/MS spectrum of the DMOX derivative of compound $\mathrm{X}$ had a molecular ion with a $m / z$ ratio of 333 and fragments of $m / z 168$ and 248, due to allylic cleavage of the fatty acid chain (Fig. 3B). The mass spectrum also exhibited differences of 12 mass
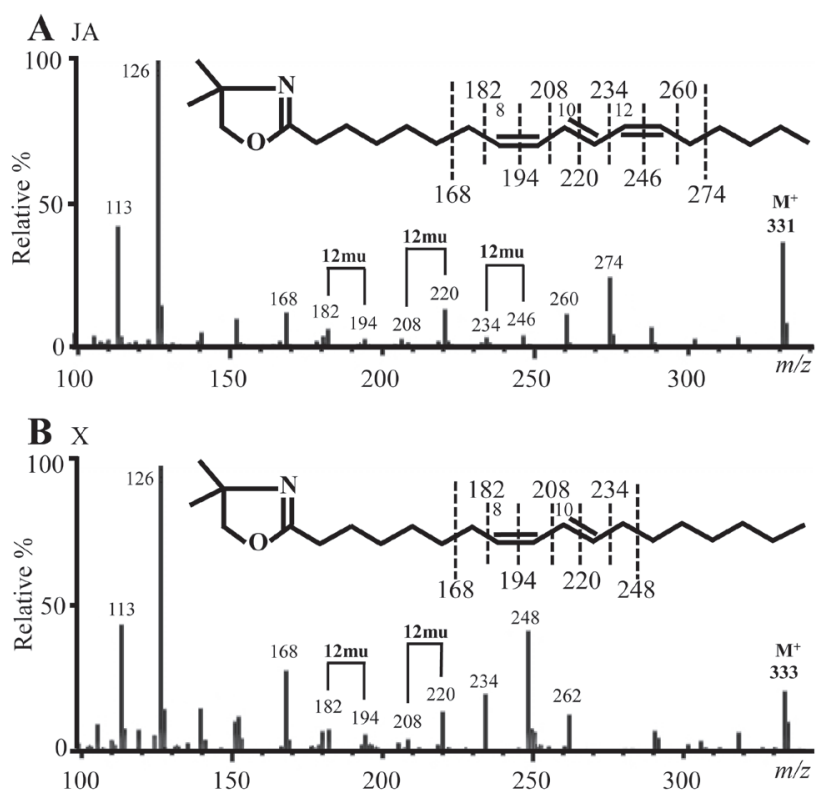

C $9 \mathrm{c}, 11 \mathrm{t}-\mathrm{CL} \mathrm{A}$

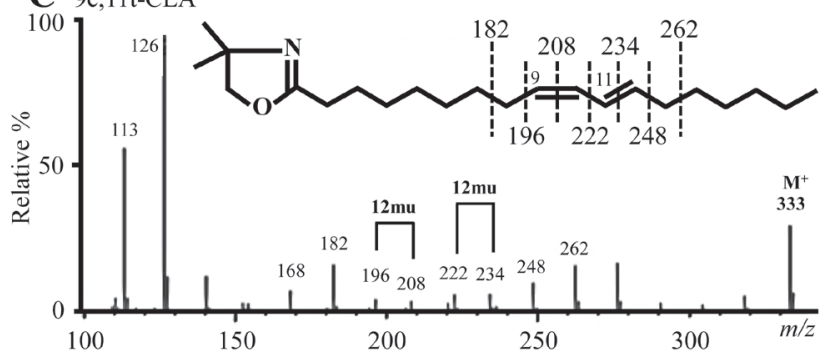

Fig. 3 GC-EI/MS spectrum of DMOX derivatives of JA (8c,10t,12c-18:3) (A), compound X (B), and 9c,11t-CLA (C). FAMEs synthesized from total lipids in the thoracic duct lymph of rats were converted to DMOX derivatives and analyzed using GC-EI/MS. 
units between fragments of $m / z 182$ and 194, and between fragments of $m / z 208$ and 220, indicating the presence of conjugated double bonds at carbon atoms 8 and 10. These results show that compound $\mathrm{X}$ was $8,10-\mathrm{CLA}$. In addition, because 8,10-CLA and 9c,11t-CLA had the same retention time, the GC-EI/MS spectrum of the DMOX derivative of compound X and 9c,11t-CLA (standard) were compared. The spectrum of the DMOX derivative of compound X differed from that of 9c,11t-CLA (Fig. 3B, C), indicating that JA was converted to a CLA through a $\Delta 12$-saturation reaction in the small intestine.

Next, the lymphatic recovery and conversion rate of JA were compared with those of $\alpha$-ESA. Since $\alpha$-ESA is converted to $9 \mathrm{c}, 11 \mathrm{t}-\mathrm{CLA}$, the lymphatic recovery of $\alpha$-ESA in TO-treated rats was determined as the total of recovered
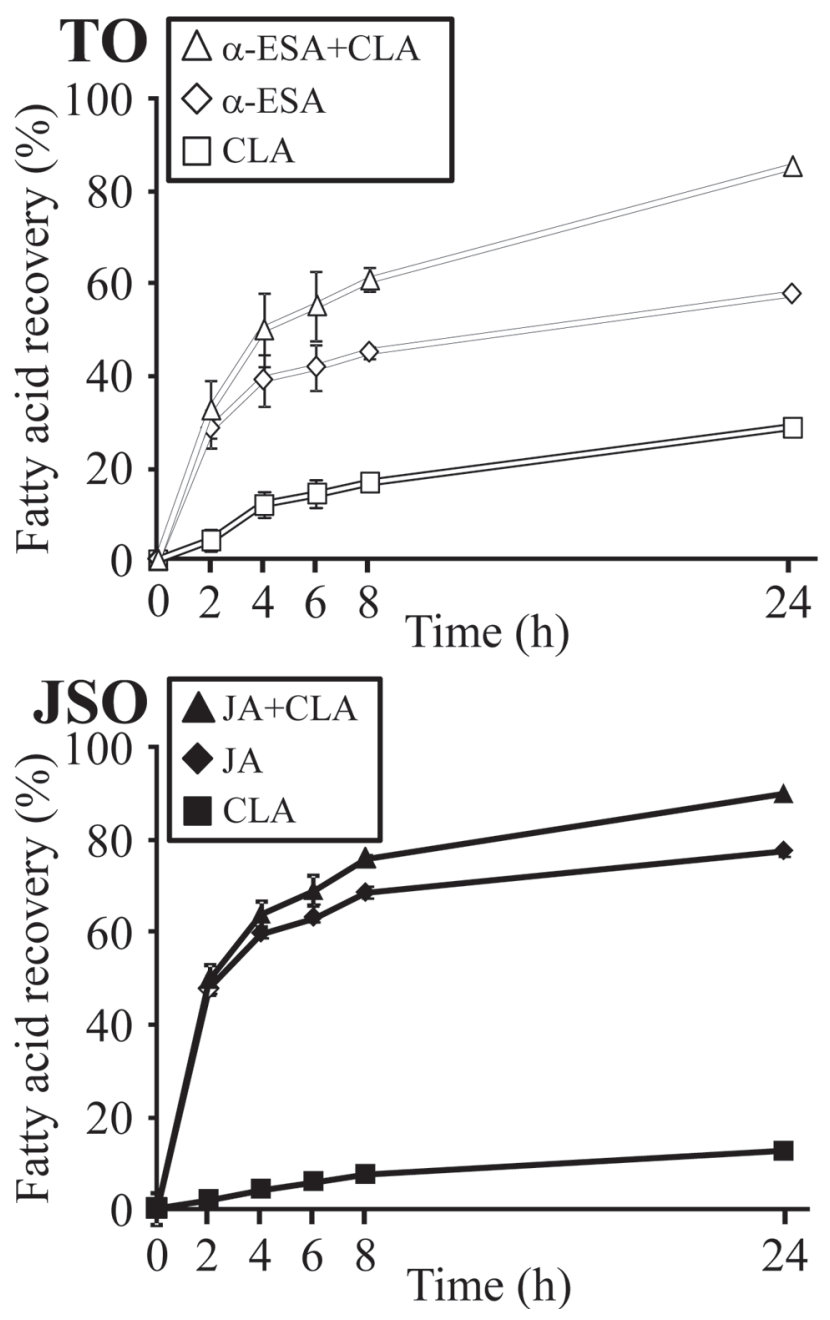

Fig. 4 Cumulative recovery of conjugated fatty acids in the thoracic duct lymph of rats infused with an emulsion containing TO or JSO $(\alpha$-ESA and 9c,11t-CLA in those infused with TO; JA and $8,10-$ CLA in those infused with JSO). Values are means \pm SE, $n=4$.
$\alpha$-ESA and 9c,11t-CLA. Based on this approach, the lymphatic recovery of $\alpha$-ESA in TO-treated rats was $62 \%$ at 8 $\mathrm{h}$ after administration and $86 \%$ after $24 \mathrm{~h}$ (Fig. 4A) : 57\% of administered $\alpha$-ESA was recovered unchanged and $29 \%$ was collected as 9c,11t-CLA due to metabolism of $\alpha$-ESA; therefore, about $34 \%$ of $\alpha$-ESA absorbed in rats was converted to 9c,11t-CLA. JA is converted to 8,10-CLA in vivo, as shown above, and therefore the lymphatic recovery of JA in JSO-treated rats was determined as the total of recovered JA and 8,11-CLA. The lymphatic recovery of JA in JSO-treated rats was $76 \%$ at $8 \mathrm{~h}$ after administration and 89\% after $24 \mathrm{~h}$ (Fig. 4B) : 77\% of administered JA was recovered unchanged and $12 \%$ was collected as 8,10-CLA; therefore, about $13 \%$ of JA absorbed in rats was converted to 8,10-CLA. Overall, the results show that most administered $\alpha$-ESA and JA was absorbed in $24 \mathrm{~h}$.

The lymphatic recoveries of $\alpha$-ESA and JA were also calculated for different time intervals (Fig. 5). There was a significant difference between recoveries of unconverted CLnAs in the JSO and TO groups for all intervals (Fig. 5A) . Recovery of unconverted JA was significantly greater than that of unconverted $\alpha$-ESA in all intervals. Overall, these results show that the absorption rate of unconverted $\alpha$-ESA was lower than that of unconverted JA. A comparison of the percentage conversion of $\alpha$-ESA and JA to CLA (Fig. 5B) also indicated a significant difference between the two groups for all intervals, with a significantly greater percentage conversion of $\alpha$-ESA in all intervals. Conversion
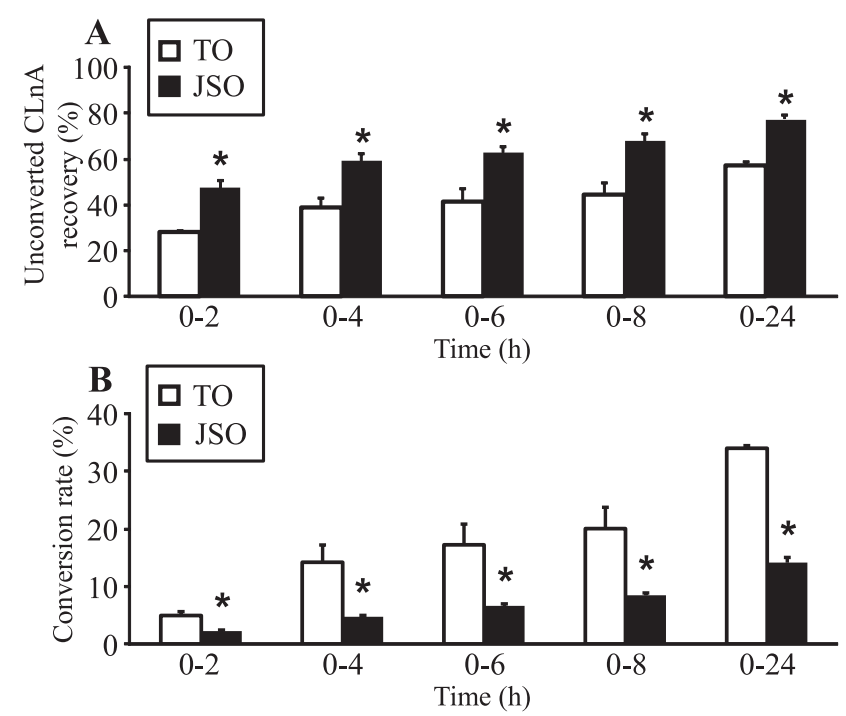

Fig. 5 Cumulative recovery of unconverted CLnAs (A) and conversion rate from a CLnA to a CLA (B) in the thoracic duct lymph of rats infused with an emulsion containing TO or JSO $(\alpha-$ ESA and $9 \mathrm{c}, 11 \mathrm{t}-\mathrm{CLA}$ in those infused with TO; JA and $8,10-C L A$ in those infused with JSO). Values are means $\pm \mathrm{SE}, \mathrm{n}=4$. ${ }^{*} p<0.05$ (vs. TO). 


\section{R. Kijima and T. Tsuduki et al.}

of $\alpha$-ESA to CLA was about two fold higher than conversion of JA, and thus unchanged JA remains at a higher concentration than unchanged $\alpha$-ESA following administration in rats.

\section{DISCUSSION}

In our previous studies, we have shown that the anti-tumor activity of CLnAs is stronger than that of CLAs and that JA has the strongest effect among CLnAs ${ }^{11,15)}$. These results suggest that JA may be a useful physiologically active substance that may have important applications in humans. Therefore, in this study we examined the absorption and metabolism of JA, with the goal of obtaining information that will permit use of JA in humans. Our results provide new findings concerning the absorption and metabolism of JA.

JA was detected in lymph of rats after administration (Fig. 2), which indicates that JA was absorbed, similarly to previous findings for other molecules with conjugated triene systems: $\alpha$-ESA and punicic acid (PA; $9 \mathrm{c}, 11 \mathrm{t}, 13 \mathrm{c}-$ $18: 3)^{13)}$. Moreover, we have previously shown that $\alpha$-ESA and PA are metabolized to 9,11-CLA in $\operatorname{rats}^{12,13)}$. In this study, we showed that JA was metabolized to 8,10-CLA based on findings in GC-EI/MS (Fig. 3) as well as $\alpha$-ESA and PA. The majority of JA administered to rats was absorbed as JA, but some JA is converted to CLA in the small intestine. This conversion reaction occurs at the double bond at the $\Delta 12$ position and is a specific reaction in fatty acid metabolism. We previously showed a similar reaction at the double bond at the $\Delta 13$ position of $\alpha$-ESA and $\mathrm{PA}^{12,13)}$. Therefore, these results suggest that the double bond distal to the carboxyl group in the conjugated triene structure is selectively saturated in this reaction.

Conversion of JA to CLA was detected two hours after administration (Fig. 2), which indicates that the conversion reaction is very fast compared with reactions such as auto-oxidation. Therefore, it is likely that this reaction is catalyzed by an enzyme in vivo for JA, as well as for $\alpha$-ESA and $\mathrm{PA}^{13,14)}$, but we currently cannot identify the enzyme. However, the $\Delta 13$-saturation reactionis probably mediated by a type of oxidoreductase, since these enzymes catalyze NADPH-dependent saturation of double bonds ${ }^{14)}$. The reaction is also similar to that in a metabolic pathway of leukotriene $\mathrm{B}_{4}\left(\mathrm{LTB}_{4}\right)$, which is an activator of leukocytes and contains a conjugated triene structure. Although $\omega$-oxidation is the major deactivation pathway of $\mathrm{LTB}_{4}$ in leukocytes, another pathway proceeding by reduction of conjugated double bonds has been reported ${ }^{20-22)}$. In the reductive pathway, $\mathrm{LTB}_{4}$ is converted to 12 -oxo-LTB 4 by $\mathrm{LTB}_{4}$ 12-hydroxydehydrogenase, which is distributed in various porcine tissues and leukocytes ${ }^{20,21)} \cdot 12$-oxo-LTB ${ }_{4}$ is then converted into 10,11,14,15-tetrahydro-12-oxo-LTB 4 (conju- gated dienoic acid) by another enzyme, which has yet to be identified ${ }^{20-22)}$. The double bond saturation reaction is also similar to that occurring in the metabolic conversion of retinol to 13,14 -dihydroretinol ${ }^{23,24)}$. Therefore, we speculate that either a novel enzyme recognizing conjugated trienoic acids or the enzyme active in the $\mathrm{LTB}_{4}$ or retinol reductive pathway may convert JA (a conjugated triene) to 8,10-CLA (a conjugated diene) through $\Delta 12$-saturation.

We identified 8,10-CLA as a metabolite of JA (Fig. 3). CLAs have useful physiologic activity ${ }^{1-5)}$, but the physiological function of 8,10-CLA is unknown. Therefore, the physiologic activity of JA might be responsible for that of 8,10-CLA. Percentage conversion of JA was lower than that of $\alpha$-ESA (Figs. 4,5 ) and we previously showed that the conversion rate of $\alpha$-ESA was higher than that of $\mathrm{PA}^{13)}$. The difference between $\alpha$-ESA and PA may be explained by the double bond configuration, since the double bond at the $\Delta 13$ position of $\alpha$-ESA is a trans isomer, whereas that of PA is cis. Thus, a trans double bond may more easily undergo enzymatic $\Delta 13$-saturation. This is consistent with the slow saturation reaction of JA, in which the double bond is in the cis configuration.

We previously showed that CLnAs have a stronger antitumor effect than CLAs in vivo and in vitro ${ }^{11)}$. This may be because CLnAs are not readily metabolized, which may also explain the strong physiological effects of JA, and it is of note that JA has a stronger anti-tumor effect than $\alpha$-ESA ${ }^{15)}$. However, the relationship between metabolic response and physiological benefits is uncertain and further work is needed to address this issue. Our results clearly show that JA is absorbed in the rat intestine more rapidly than $\alpha$-ESA (Figs. 4,5 ) and we have previously shown that the rate of absorption of $\alpha$-ESA was slower than that of $\alpha$-linolenic acid $(9 c, 12 c, 15 c-18: 3)^{13)}$. We have also shown that JA is absorbed in cultured cells faster than $\alpha$-ESA ${ }^{15)}$. These differences may be related to the polarity of the molecules, with JA showing faster absorption because the polarity of JA is lower than that of $\alpha$-ESA.

In this study, the percentage of JA in jacaranda seed oil was different compared with that of $\alpha$-ESA in tung oil (Table 1). The difference of fatty acid levels in the emulsion has the possibility of influencing the lymphatic recovery. However, we previously showed that the difference of CLA levels in the emulsion did not give the substantial change to the lymphatic recovery of CLA ${ }^{16)}$. Therefore, it was thought that there was no big influence in the lymphatic recovery of conjugated fatty acids in dosage of this experiment (200 mg triacylglycerol/rat).

\section{CONCLUSION}

In this study, we examined the absorption and metabolism of JA. We showed that JA is rapidly absorbed and con- 
verted to a CLA in rats and that the percentage conversion of JA is lower than that of $\alpha$-ESA. This is the first report on the absorption and metabolism of JA and this information is important for use of JA as a functional food. However, further studies of the absorption and metabolism of JA are required to resolve several remaining questions.

\section{ACKNOWLEDGMENT}

This study was supported by KAKENHI (20780099) of JSPS, Japan. The authors declare that there are no conflicts of interest.

\section{References}

1) Ha, Y. L.; Grimm, N. K.; Pariza, M. W. Anticarcinogens from fried ground beef: heat-altered derivatives of linoleic acid, Carcinogenesis 8, 1881-1887(1987).

2) Lee, K. N.; Kritchevsky, D.; Pariza, M. W. Conjugated linoleic acid and atherosclerosis in rabbits, Atherosclerosis 108, 19-25 (1994).

3) Park, Y.; Albright, K. J.; Storkson, J. M.; Liu, W.; Cook, M. E.; Pariza, M. W. Changes in body composition in mice during feeding and withdrawal of conjugated linoleic acid, Lipids 34, 243-248 (1999).

4) Ip, C.; Chin, S. F.; Scimeca, J. A.; Pariza, M. W. Mammary tumor prevention by conjugated dienoic derivative of linoleic acid, Cancer Res. 51, 6118-6124 (1991).

5) Igarashi, M.; Miyazawa, T. The growth inhibitory effect on conjugated linoleic acid on a human hepatoma cell line, HepG2, is induced by a change in fatty acid metabolism, but not the facilitation of lipid peroxidation in the cells, Biochem. Biophys. Acta 1530, 162-171 (2001).

6) Larsen, T. M.; Toubro, S.; Astrup, A. Efficacy and safety of dietary supplements containing CLA for the treatment of obesity: evidence from animal and human studies, J. Lipid Res. 44, 2234-41 (2003).

7) Gaullier, J. M.; Halse, J.; Hoye, K.; Kristiansen, K.; Fagertun, H.; Vik, H.; Gudmundsen, O. Supplementation with conjugated linoleic acid for 24 months is well tolerated by and reduces body fat mass in healthy, overweight humans, J. Nutr. 135, 778-84 (2005).

8) Badami, R. C.; Patil, K. B. Structure and occurrence of unusual fatty acids in minor seed oils, Prog. Lipid Res. 19, 119-153 (1980).

9) Joh, Y. G.; Kim, S. J.; Christie, W. W. The structure of the triacylglycerols, containing punicic acid, in the seed oil of Trichosanthes kirilowii, J. Am. Oil Chem. Soc. 72, 1037-1042 (1995).

10) Hopkins, Y. Fatty acids with conjugated unsaturation,
In: Topics in Lipid Chemistry (Gunstone, F. D., ed.), ELEK Science, London, pp. 37-87(1972).

11) Tsuzuki, T.; Tokuyama, Y.; Igarashi, M.; Miyazawa, T. Tumor growth suppression by alpha-eleostearic acid, a linolenic acid isomer with a conjugated triene system, via lipid peroxidation, Carcinogenesis 25, 1417-25 (2004).

12) Tsuduki, T.; Igarashi, M.; Komai, M.; Miyazawa, T. The metabolic conversion of 9,11,13-eleostearic acid (18:3) to 9,11-conjugated linoleic acid (18:2) in the rat, $J$. Nutri. Sci. Vitaminol. 49, 195-200 (2003).

13) Tsuzuki, T.; Kawakami, Y.; Abe, R.; Nakagawa, K.; Koba, K.; Imamura, J.; Iwata, T.; Ikeda, I.; Miyazawa, T. Conjugated linolenic acid is slowly absorbed in rat intestine, but quickly converted to conjugated linoleic acid, J. Nutr. 136, 2153-9 (2006) .

14） Tsuzuki, T.; Tokuyama, Y.; Igarashi, M.; Nakagawa, K.; Ohsaki, Y.; Komai, M.; Miyazawa, T. $\alpha$-Eleostearic acid (9Z11E13E-18:3) is quickly converted to conjugated linoleic acid (9Z11E-18:2) in rats, J. Nutr. 134, 2634-9 (2004).

15) Shinohara, N.; Tsuduki, T; Ito, J.; Honma, T.; Kijima, R.; Sugawara, S.; Arai, T.; Yamasaki, M.; Ikezaki, A.; Yokoyama, M.; Nishiyama, K.; Nakagawa, K.; Miyazawa, T.; Ikeda, I. Jacaric acid, a linolenic acid isomer with a conjugated triene system, has a strong antitumor effect in vitro and in vivo, Biochim. Biophys. Acta 1821, 980-8(2012).

16) Tsuzuki, T.; Ikeda, I. Slow absorption of conjugated linoleic acid in rat intestines, and similar absorption rates of 9c,11t-conjugated linoleic acid and 10t,12cconjugated linoleic acid, Biosci. Biotechnol. Biochem. 71, 2034-40 (2007).

17) Bligh, E. G.; Dyer, W. J. A rapid method of total lipid extraction and purification, J. Biochem. Physiol. 37, 911-7 (1959).

18) Igarashi, M.; Tsuzuki, T.; Kambe, T.; Miyazawa, T. Recommended methods of fatty acid methylester preparation for conjugated dienes and trienes in food and biological samples, J. Nutr. Sci. Vitaminol. 50, 121-8 (2004).

19) Sato, K.; Shinohara, N.; Honma, T.; Ito, J.; Arai,T.; Nosaka, N.; Aoyama, T.; Tsuduki, T.; Ikeda, I. The change in conjugated linoleic acid concentration in blood of Japanese fed a conjugated linoleic acid diet, J. Nutri. Sci. Vitaminol. 57, 364-71(2011).

20） Yokomizo, T.; Izumi, T.; Takahashi, T.; Kasama, T.; Kobayashi, Y.; Sato, F.; Taketani, Y.; Shimizu, T. Enzymatic inactivation of leukotriene $\mathrm{B}_{4}$ by a novel enzyme found in the porcine kidney. Purification and properties of leukotriene $\mathrm{B}_{4}$ 12-hydroxydehydrogenase, $J$. Biol. Chem. 268, 18128-35(1993).

21) Yokomizo, T.; Uozumi N.; Takahashi, T.; Kume, K.; Izumi, T.; Shimizu, T. Leukotriene $\mathrm{A}_{4}$ hydroxylase and 


\section{R. Kijima and T. Tsuduki et al.}

leukotriene $\mathrm{B}_{4}$ metabolism, J. Lipid Mediat. Cell Signal. 12, 321-32 (1995).

22) Wainwright, S. L.; Powell, W. S. Mechanism for the formation of dihydro metabolites of 12-hydroxyeicosanoids. Conversion of leukotriene $\mathrm{B}_{4}$ and 12-hydroxy-5, 8, 10, 14-eicosatetraenoic acid to 12-oxo intermediates, J. Biol. Chem. 266, 20899-906 (1991).

23) Moise, A. R.; Kuksa, V.; Imanishi, Y.; Palczewski, K.
Identification of all-trans-retinol: all-trans-13,14-dihydroretinolsaturase, J. Biol. Chem. 279, 50230-42 (2004).

24) Moise, A. R.; Isken, A.; Domínguez, M.; de Lera, A. R.; von Lintig, J.; Palczewski, K. Specificity of zebrafish retinol saturase: formation of all-trans-13,14-dihydroretinol and all-trans-7,8-dihydroretinol, Biochemistry 46, 1811-20 (2007). 